\title{
Natriuretic peptides: Prediction of cardiovascular disease in the general population and high risk populations
}

\author{
Per Hildebrandt* \\ Section of Cardiology, Glostrup University Hospital, Denmark
}

\begin{abstract}
The natriuretic peptides, especially the B-type peptide (BNP) and its inactive split-product N-terminal proBNP (NtproBNP) are increasingly used in screening for heart failure, primarily with reduced systolic function, in patients with symptoms suggestive of heart failure, as well in the stable (General Practitioner) setting as in the acute setting.

Supporting this use is a very strong prognostic value of the natriuretic peptides. This has been shown in as well heart failure as acute coronary syndromes, but also in the general population and in high-risk groups as patients with diabetes, hypertension and coronary artery disease.

This has of course raised interest for the use of the natriuretic peptides as a risk marker and for screening for heart failure with reduced systolic function in these populations. In symptomatic persons and in high risk populations, the natriuretic peptides have demonstrated a high sensitivity for ruling out the disease, if the right decision limits are choosen. Thus the number of normal echocardiographies can be reduced.

More recently, the use in screening asymptomatic persons for left ventricular systolic dysfunction has gained more interest. In the unselected population, screening would probably not be cost-effective. In populations with a higher pre-test probability for heart failure, as patients with diabetes, hypertension and stable coronary artery disease, screening would presumably be more cost-effective, but evidence for the use in this setting is still lacking.
\end{abstract}

Keywords: Brain natriuretic peptide, cardiovascular disease, general population, prognosis, heart failure

\section{Introduction}

The use of biomarkers, including the natriuretic peptides, in predicting cardiovascular disease has been gaining increasing interest during recent years. Several applications for the use of biomarkers have been suggested in the general population and in high-risk groups such as those with coronary artery disease, diabetes and hypertension. Initially, biomarkers were used or suggested primarily for use as a prognostic tool, but more recently some have shown promise also in diagnosing or screening for disease.

*Per Hildebrandt, Section of Cardiology, Glostrup University Hospital, Nordre Ringvej, DK-2650 Glostrup, Denmark. Tel.: +45 27 1354 26; E-mail: p.hildebrandt@dadlnet.dk.
Presumably, some of the most promising biomarkers at present are the natriuretic peptides. These have been known and studied for many years but have only recently drawn increasing interest thanks to much easier access to commercially available assays. Initially, most studies were carried out on atrial natriuretic peptide (ANP) (then termed atrial natriuretic factor, ANF). Unfortunately ANP and the other main natriuretic peptide BNP were fairly unstable and difficult to measure. The emergence of much easier assays for the B-type natriuretic peptides (BNP and its inactive split product NTproBNP) enabled larger studies demonstrating their strong prognostic and clinical value. More recently new techniques of measuring proANP have made it more accessible, early results have shown it to be a strong prognostic marker [1] and further work is in 
progress to demonstrate its clinical value, perhaps in combination with the B-type natriuretic peptides.

The prehormone preproBNP is secreted in the myocardium, split to proBNP and again to the active BNP and the inactive split product NTproBNP. The half-life of BNP is short (approximately $18 \mathrm{~min}$ ), while the halflife of NT-pro- BNP is substantially longer. In response to myocardial wall stress, BNP is secreted from the myocardial cell predominantly in the left ventricle and to a lesser degree from the atria and right ventricle. Diseases that impact wall stress, especially heart failure, induce an increase - most often high - in B-type natriuretic peptides [2]. BNP and NT-proBNP are fairly similar in their response to different stimuli, but NTproBNP is present in higher concentrations, is more stable at room temperature and can be sent as whole blood by ordinary mail to be centrifuged and analysed within 2 days. Thus BNP and NT-proBNP would in general be equally applicable in hospital settings, while NT-proBNP may be superior in an outpatient or general practitioner setting.

\section{Prognostic value of the B-type natriuretic peptides}

The first studies carried out in stable heart failure patients showed a very strong prognostic value of BNP and NTproBNP, much higher than that of traditional risk markers such as left ventricular systolic function (ejection fraction) and New York Heart Association class [3]. Since then the strong prognostic value in stable heart failure has been well established, and also extended to other cardiovascular disease entities as acute heart failure [4], acute [5] and chronic [6] coronary artery disease, and the general population and high risk populations.

\subsection{Prognostic value in the general population}

Many different biochemical markers have for many years been known to be cardiovascular risk markers in the general population. The strongest were probably microalbuminuria [7,8] and, among the plasma markers, high sensitivity C-reactive protein (hsCRP) [9]. More recently a number of studies have shown a strong prognostic value for the B-type natriuretic peptides in predicting mortality as well as hospitalization for heart failure, stroke and atrial fibrillation, but only a fairly weak predictive value for myocardial infarction.
Wang et al. [10] reported powerful independent associations among BNP and death and cardiovascular events in the Framingham Offspring Study. This finding was of particular interest in light of the previous report of an only modest utility of BNP testing to screen for LVSD and LVH in this same cohort [11]. In a population-based prospective study of individuals aged 50-89 years [12], NT-proBNP, hsCRP, and urinary albumin/creatinine measurements were performed in 626 participants and correlated with events through 5 years of follow-up. After adjustment for cardiovascular risk factors and serum creatinine, NT-proBNP levels $\geqslant$ the 80th percentile were associated with an approximate 2-fold increase in the risk for mortality and a 3.24-fold increased risk for first major cardiovascular events. Of particular importance, the association with mortality persisted after further adjustment for LVSD. The prognostic information provided by NT proBNP was greater than that provided by hsCRP. In the Olmsted County population of 1,991 subjects without HF and with complete clinical and echocardiographic data, NT-proBNP as well as BNP predicted mortality through 5.6 years of follow-up, after adjustment for traditional risk factors and echocardiographic abnormalities [13]. In a recent paper we demonstrated that the additional use of biomarkers would change the HeartScore classification significantly in one-fifth of a general population [14].

In 2,656 persons from the Danish Glostrup population studies observed for 9.4 years, serum NT-proBNP and urinary albumin/ creatinine, but not hsCRP, predicted cardiovascular death in an additive fashion after adjustment for established cardiovascular risk factors [15]. In contrast to this finding, data from Sweden on 1,135 elderly men shoved an improvement in risk stratification for cardiovascular death using several biomarkers (troponin I, NT-proBNP, cystatin C and hsCRP) [16]. At present, the clinical superiority of a multimarker strategy over the use of only a single marker could be promising, but further investigations on this are needed.

In conclusion, BNP and NT-proBNP have shown very strong prognostic value in the general population. Data indicate that the use in risk evaluation could result in an improved risk classification, but confirmatory data supporting a use for this is still lacking.

\subsection{Prognostic value in high risk populations}

It must be assumed that BNP and NT-proBNP will have the same strong prognostic value in high risk populations as in the general population, identifying sub- 
groups in a high risk group with an even higher risk. The high risk groups primarily studied are stable coronary artery disease, diabetes mellitus and hypertension.

No specific outcome study in a mixed high risk population has been published. Results from the large Heart Outcomes Prevention Evaluation (HOPE) study [17] and the Heart Protection Study (HPS) [18] involving high-risk patients primarily with established ischemic heart disease, showed that NT-proBNP was predictive of cardiovascular events and highly predictive for the development of heart failure. The highest NT-proBNP quintile in the HPS had an adjusted relative risk for heart failure hospitalization or death that was 9 times greater than the lowest quintile.

\subsubsection{Stable coronary artery disease}

NT-proBNP has been shown to be a strong prognostic marker in patients with stable coronary heart disease $[6,19]$. In one study 1,034 patients referred to elective coronary angiography because of symptoms or signs of coronary heart disease were studied [6]. The level of NT-proBNP was significantly higher in patients with severe angiographic coronary disease as compared to patients with no coronary disease or minor atherosclerotic lesions at angiography. Patients were followed up for a median of 9 years with registration of survival status. A significant decreased survival with increasing levels of NT-proBNP were found. The hazard ratio for death from any cause was twice as high in patients with NT-proBNP levels in the highest quartile as compared to patients with NT-proBNP levels in the lowest quartile. This increased risk was independent of conventional cardiovascular risk factors and invasive measurements of severity of coronary disease and left ventricular dysfunction.

\subsubsection{Diabetes mellitus}

Individuals with diabetes generally have slightly higher levels of NT-proBNP and BNP in comparison to people without diabetes [20]. Several studies have shown a very strong prognostic value of NTproBNP in patients with type 1 [21] and type 2 diabetes [22,23]. Even among diabetic patients with micro- or macroalbuminuria, the strong prognostic value is preserved and is supplementary to that conferred by micro- or macroalbuminuria alone. For example, in one cohort of diabetic patients with long-term followup, the 9-year mortality in those with macroalbuminuria and NT-proBNP above the median, macroalbuminuria and NT-proBNP below the median and in those without albuminuria was $40 \%, 12 \%$ and $7 \%$, respective- ly [21]. In patients with microalbuminuria, compared to those with normoalbuminuria, a steeper increase in NT-proBNP is seen over time in parallel with a more severe prognosis. In diabetic out-patients, NT-proBNP has a very high negative predictive value for cardiovascular events, thus identifying individuals who are at lower risk for cardiovascular events [24].

\subsubsection{Hypertension}

NT-proBNP is a strong predictor for both mortality and morbidity in patients with hypertension. The Losartan Intervention for Endpoint Reduction in Hypertension (LIFE) study recruited high-risk patients with hypertension and electrocardiographic LVH [25]. NT-proBNP testing markedly improved risk stratification when added to the presence or absence of a history of cardiovascular disease (CVD), implying an incremental usefulness for risk stratification as the medical complexity of the patient increased.

\section{Clinical use}

The very strong prognostic value of BNP and NTproBNP has given rise to a substantial interest in the clinical use of these markers. At present the primary approved use is in screening for heart failure in symptomatic outpatients [26] and in patients admitted to hospital for acute heart failure [27].

Other applications, e.g. screening for heart failure and/or risk in the general population or in high-risk populations such as individuals with diabetes, may be of interest but have not been conclusively established. Furthermore, studies are currently underway to test the utility of these markers in guiding therapy and improving control in outpatients with heart failure.

\subsection{Screening symptomatic individuals in the general population for heart failure}

Identification of both frank heart failure and asymptomatic left ventricular systolic dysfunction (LVSD) is crucial, as the untreated prognosis is very severe, but modern investigation techniques along with the introduction of proven pharmacological and mechanical treatments markedly reduce morbidity and mortality [28]. As the symptoms of heart failure are often relatively non-specific, there is a risk that patients may not be properly investigated, with a resulting delay (or failure) of diagnosis and institution of appropriate therapy. Following a comprehensive history and physi- 
cal examination, the most commonly used diagnostic method for outpatient evaluation of cardiac function is echocardiography, but this investigation demands expensive equipment and trained sonographers. Thus, a marker for heart failure among symptomatic outpatients is very valuable, and in recent years the use of B-type natriuretic peptides has been implemented in European guidelines as part of the screening process for symptomatic outpatients [28].

Several studies have been performed to test the utility of BNP and NT-proBNP in evaluating patients with symptoms suggestive of heart failure, each demonstrating superb negative predictive values and sensitivities of approximately $99 \%$ and $90 \%$, respectively. On the basis of these studies, the accepted 'rule-out' value of NT-proBNP is $125 \mathrm{pg} / \mathrm{ml}$ [26], regardless of age and gender. The very high sensitivity implies a very low risk of overlooking a patient with heart failure, a fact essential for effective screening. Due to the normal values increasing with age, the specificity in the elderly is relatively low using this value, and work on establishing age specific values are in progress.

\subsection{Putative pitfalls in the use of BNP and NT-proBNP}

There are several putative pitfalls in the use of the Btype natriuretic peptides in screening for heart failure, as several factors influence their levels. Some drugs, especially angiotensin-converting enzyme inhibitors and angiotensin II receptor blockers, but also diuretics and spironolactone, reduce B-type peptide levels. Obesity and hypothyroidism also have the same effect on the B-type natriuretic peptides, but present experience suggests that the decrease due to drugs and obesity is modest, and the risk of inducing false-negative values, and thus overlooking heart failure, is very small. Other factors induce an increase in the levels of Btype natriuretic peptides, the major problem being the higher concentrations seen with increasing age in individuals without clinically overt cardiac disease [29, 30]. The reason for this could be age-related changes in the metabolism of B-type natriuretic peptides, agedependent changes in the heart, or it could be due to the decrease in renal function with age. Other factors that induce an increase in B-type natriuretic peptides are impaired renal function (primarily at least moderate impairment), valve diseases, atrial fibrillation and left ventricular hypertrophy. The increased risk of falsepositive values in these situations is no problem in clinical practice, as further echocardiographic investigation would often be warranted in these conditions. Gender also affects BNP and NT-proBNP levels, and higher levels are found in women than in men [29,30].The magnitude of the differences is fairly small and of no importance in outpatient testing and the same cut-off value may be used in both sexes. A substantial proportion of heart failure patients have non-systolic heart failure, including diastolic dysfunction. The value of Btype natriuretic peptides in evaluating heart failure in these patients has been less well investigated, partly due to the lack of diagnostic gold standards for nonsystolic heart failure. From the published literature it is reassuring that BNP [31] and NT-proBNP [32] appear useful for evaluating patients with non-systolic heart failure, although generally lower values in the context of nonsystolic heart failure are expected.

\subsection{Screening asymptomatic individuals in the general population for heart failure}

Screening is only warranted if a positive finding has any clinical consequence. The detection of asymptomatic left ventricular systolic dysfunction (LVSD) would have clinical implications because treatment with angiotensin-converting enzyme inhibitors would be indicated.

Several studies have shown that the B-type natriuretic peptides are of value in detecting asymptomatic LVSD in the general population [29,33]. However, the prevalence of heart failure (the pre-test probability) is so low that screening using B-type peptides, followed by further investigation with echocardiography in patients with elevated values, would lead to a huge amount of normal echocardiographic test results in order to detect those very few patients with asymptomatic LVSD [34]. Screening in the general population would probably not be cost-effective, but it is likely that the higher prevalence of LVSD in high risk patients, e.g. those with diabetes, hypertension or ischemic heart disease, would increase the cost-effectiveness to an acceptable level [34]. However, definitive studies evaluating such targeted application of B-type peptides have not been performed.

\subsection{Screening asymptomatic high risk individuals}

\subsubsection{Stable coronary artery disease patients}

These comprise a high-risk group with putative indication for the use of BNP/NT-proBNP as a screening tool for left ventricular systolic dysfunction [34]. Studies indicate that the B-type natriuretic peptides are of 
limited value in detecting left ventricular systolic dysfunction in this group, presumably because of a higher prevalence of competing diagnoses including ischemia, left ventricular hypertrophy, and diastolic dysfunction, which all may cause elevations in natriuretic peptide levels [35,36].

Recently, several studies have confirmed the strong epidemiological association between the B-type natriuretic peptides and severity of coronary atherosclerotic lesions and signs of ischemia. This has led to the assumption that ischemia per se contributes significantly to circulating levels of natriuretic peptides, and this may be one of the reasons to the adverse prognosis in these patients. However, whether natriuretic peptides will prove effective in diagnosis of coronary disease remains yet to be established, but available work is not encouraging [37,38].

\subsubsection{Diabetes mellitus}

The B-type natriuretic peptides may be useful for screening in the diabetic population, for example at a diabetes outpatient clinic, to detect those with 'asymptomatic' heart failure. The prevalence of heart failure in this group is substantially higher than among non-diabetic subjects [39], thus making screening substantially more cost-effective. No studies on costeffectiveness have been performed in diabetic patients, but Nielsen et al. [34] analysed a population-based study from Scotland and found that the number of patients who would need to be examined with echocardiography (after NTproBNP screening) in order to identify one patient with LVSD was 67 in the general population, which was reduced to 12 in a high-risk population with either hypertension or electrocardiographic changes but without heart failure. Although these figures could make screening a much more attractive proposition, a major problem is the lack of data on diabetes and the lack of validated cut-off values as those existing for symptomatic patients. Therefore, more widespread use of this indication should await further clarification. Another interesting application would be screening for coronary artery disease in asymptomatic patients with diabetes. Only sparse and not too encouraging studies on this are published, but further studies are ongoing.

\subsubsection{Hypertension}

In population studies, the natriuretic peptide levels are generally higher in patients with hypertension than in normotensives, and levels of NT-proBNP are related to both clinic and 24-hour ambulatory blood pressures [40]. Furthermore, NT-proBNP is related to left ventricular mass measured by magnetic resonance imaging [41], although the elevation in NT-proBNP with left ventricular hypertrophy (LVH) is generally less pronounced than in patients with significant LVSD. Work from the Framingham study shows that the value of general community screening with BNP for LVH is modest and cannot be recommended [11]. There are no studies of the utility of BNP or NT-proBNP for detection of LVSD conducted solely within hypertensive populations, but as mentioned above, Nielsen et al found this approach cost-effective. Although there is a sound rationale behind such an approach, further prospective observational data are required.

\section{Conclusions}

The B-type natriuretic peptides (BNP and NTproBNP) have an established use in screening patients with symptoms suggestive of chronic heart failure before further investigation (echocardiography) and for risk stratification in acute heart failure.

The uniform demonstration of the B-type natriuretic peptides very strong prognostic value in all populations studied gives hope for the use as a risk marker in different populations, but primarily as a screening marker for asymptomatic left ventricular dysfunction in high risk populations.

\section{References}

[1] A. Gegenhuber, J. Struck, B. Dieplinger et al., Comparative evaluation of B-type natriuretic peptide, midregional proA-type natriuretic peptide, mid-regional pro-adrenomedullin, and Copeptin to predict 1-year mortality in patients with acute destabilized heart failure, J Card Fail 13(1) (2007), 42-49.

[2] J.P. Gøtze, Biochemistry of proBNP-derived peptides in plasma: the endocrine heart revisited, Clin Chem $\mathbf{5 0}$ (2004), 15031510 .

[3] F. Hartmann, M. Packer, A.J. Coats et al., Prognostic impact of plasma N-terminal pro-brain natriuretic peptide in severe chronic congestive heart failure: a substudy of the Carvedilol Prospective Randomized Cumulative Survival (COPERNICUS) trial, Circulation 110 (2004), 178.

[4] J.L. Januzzi, R. van Kimmenade, J. Lainchbury et al., NTproBNP testing for diagnosis and short-term prognosis in acute destabilized heart failure: an international pooled analysis of 1256 patients: the International Collaborative of NT-proBNP Study, Eur Heart J 27 (2006), 330-337.

[5] S.K. James, B. Lindahl, A. Siegbahn et al., N-terminal probrain natriuretic peptide and other risk markers for the separate prediction of mortality and subsequent myocardial infarction in patients with unstable coronary artery disease. A Global Utilization of Strategies to Open Occluded Arteries (GUSTO)-IV substudy, Circulation 108 (2003), 275-281. 
[6] C. Kragelund, B. Gronning, L. Kober et al., N-terminal proB-type natriuretic peptide and long-term mortality in stable coronary heart disease, N Engl J Med 352(7) (2005), 666-675.

[7] K. Borch-Johnsen, B. Feldt-Rasmussen, S. Strandgaard et al., Urinary albumin excretion. An independent predictor of ischaemic heart disease, ArteriosclerThrombVasc Biol 19 (1999), 1992-1997.

[8] M.H. Olsen, T.W. Hansen, M.K. Christensen et al., N-terminal pro-brain natriuretic peptide, but not high sensitivity Creactive protein, improves cardiovascular risk prediction in the general population, Eur Heart J 28(11) (2007), 1374-1381.

[9] P.M. Ridker, C.H. Hennekens, J.E. Buring and N. Rifai, Creactive protein and other markers of inflammation in the prediction of cardiovascular disease in women, $N$ Engl J Med 342(12) (2000), 836-843.

[10] T.J. Wang, M.G. Larson, D. Levy et al., Plasma natriuretic peptide levels and the risk of cardiovascular events and death, $N$ Engl J Med 350 (2004), 655-663.

[11] R.S. Vasan, E.J. Benjamin, M.G. Larson et al., Plasma natriuretic peptides for community screening for left ventricular hypertrophy and systolic dysfunction: the Framingham heart study, JAMA 288 (2002), 1252-1259.

[12] C. Kistorp, I. Raymond, F. Pedersen et al., N-terminal probrain natriuretic peptide, C-reactive protein, and urinary albumin levels as predictors of mortality and cardiovascular events in older adults, JAMA 293 (2005), 1609-1616.

[13] P.M. McKie, R.J. Rodeheffer, A. Cataliotti et al., Aminoterminal pro-B-type natriuretic peptide and B-type natriuretic peptide: biomarkers for mortality in a large community-based cohort free of heart failure, Hypertension 47 (2006), 874-880.

[14] M.H. Olsen, T.W. Hansen, M.K. Christensen et al., New risk markers may change the HeartScore risk classification significantly in one-fifth of the population, J Hum Hypertension 23(2) (2009), 105-112.

[15] M.H. Olsen, T.W. Hansen, M.K. Christensen et al., N-terminal pro-brain natriuretic peptide, but not high sensitivity Creactive protein, improves cardiovascular risk prediction in the general population, Eur Heart J 28 (2007), 1374-1381.

[16] B. Zethelius, L. Berglund, J. Sundstrom et al., Use of multiple biomarkers to improve the prediction of death from cardiovascular causes, N Engl J Med 538(20) (2008), 2107-2116

[17] S. Blankenberg, M.J. McQueen, M. Smieja et al., Comparative impact of multiple biomarkers and N-Terminal pro-brain natriuretic peptide in the context of conventional risk factors for the prediction of recurrent cardiovascular events in the Heart Outcomes Prevention Evaluation (HOPE) Study, Circulation 114 (2006), 201-208.

[18] J.R. Emberson, L.L. Ng, J. Armitage et al., N-terminal ProB-type natriuretic peptide, vascular disease risk, and cholesterol reduction among 20,536 patients in the MRC/BHF heart protection study, J Am Coll Cardiol 49 (2007), 311-319.

[19] T. Omland, M.S. Sabatine, K.A. Jablonski et al., Prognostic value of B-type natriuretic peptides in patients with stable coronary artery disease: the PEACE Trial, J Am Coll Cardiol 50(3) (2007), 205-214

[20] M. Magnusson, O. Melander, B. Israelsson et al., Elevated plasma levels of NT-proBNP in patients with type 2 diabetes without overt cardiovascular disease, Diabetes Care 27(8) (2004), 1929-1935.

[21] L. Tarnow, P. Hildebrandt, B.V. Hansen et al., Plasma Nterminal pro-brain natriuretic peptide as an independent predictor of mortality in diabetic nephropathy, Diabetologia 48 (2005), 149-155.
[22] L. Tarnow, M.A. Gall, B.V. Hansen et al., Plasma N-terminal pro-B-type natriuretic peptide and mortality in type 2 diabetes, Diabetologia 49(10) (2006), 2256-2262.

[23] P. Gaede, P. Hildebrandt, G. Hess et al., Plasma N-terminal pro-brain natriuretic peptide as a major risk marker for cardiovascular disease in patients with type 2 diabetes and microalbuminuria, Diabetologia 48 (2005), 156-163.

[24] M. Huelsmann, S. Neuhold, G. Strunk et al., NT-proBNP has a high negative predictive value to rule-out short-term cardiovascular events in patients with diabetes mellitus, Eur Heart J 29(18) (2008), 2259-2264.

[25] M.H. Olsen, K. Wachtell, O.W. Nielsen et al., N-terminal brain natriuretic peptide predicted cardiovascular events stronger than high-sensitivity C-reactive protein in hypertension: a LIFE substudy, J Hypertens 24 (2006), 1531-1539.

[26] P. Hildebrandt and P.O. Collinson, Amino-Terminal Pro-BType Natriuretic Peptide Testing to Assist the Diagnostic Evaluation of Heart Failure in Symptomatic Primary Care Patients, Am J Cardiol 101(suppl) (2008), 25A-28A.

[27] J.L. Januzzi, A.A. Chen-Tournoux and G. Moe, AminoTerminal Pro-B-Type Natriuretic Peptide Testing for the Diagnosis or Exclusion of Heart Failure in Patients with Acute Symptoms, Am J Cardiol 101(suppl) (2008), 29A-38A.

[28] K. Dickstein, A. Cohen-Solal, G. Filippatos et al., ESC Guidelines for the diagnosis and treatment of acute and chronic heart failure 2008: the Task Force for the Diagnosis and Treatment of Acute and Chronic Heart Failure 2008, Eur Heart J 29 (2008), 2388-2442.

[29] I. Raymond, B.A. Groenning, P.R. Hildebrandt et al., The influence of age, sex and other variables on the plasma level of $\mathrm{N}$-terminal pro brain natriuretic peptide in a large sample of the general population, Heart 89(7) (2003), 745-751.

[30] L.C. Costello-Boerrigter, G. Boerrigter, M.M. Redfield et al., Amino-terminal pro-B-type natriuretic peptide and B-type natriuretic peptide in the general community: determinants and detection of left ventricular dysfunction, J Am Coll Cardiol 47 (2006), 345-353.

[31] G.S. Mak, A. DeMaria, P. Clopton et al., Utility of Bnatriuretic peptide in the evaluation of left ventricular diastolic function: comparison with tissue Doppler imaging recordings, Am Heart J 148(5) (2004), 895-902.

[32] C. Tschope, M. Kasner, D. Westermann et al., The role of NT-proBNP in the diagnostics of isolated diastolic dysfunction: correlation with echocardiographic and invasive measurements, Eur Heart J 26 (2005), 2277-2284.

[33] B.A. Groenning, I. Raymond, P.R. Hildebrandt et al., Diagnostic and prognostic evaluation of left ventricular systolic heart failure by plasma N-terminal pro-brain natriuretic peptide concentrations in a large sample of the general population, Heart 90(3) (2004), 297-303.

[34] O.W. Nielsen, T.A. McDonagh, S.D. Robb et al., Retrospective analysis of the cost-effectiveness of using plasma brain natriuretic peptide in screening for left ventricular systolic dysfunction in the general population, $J$ Am Coll Cardiol 41 (2003), 113-120.

[35] K. Bibbins-Domingo, M. Ansari, N.B. Schiller et al., Is Btype natriuretic peptide a use-ful screening test for systolic or diastolic dysfunction in patients with coronary disease? Data from the Heart and Soul Study, Am J Med 116(8) (2004), 509-516.

[36] T. Omland, A. Aakvaag and H. Vik-Mo, Plasma cardiac natriuretic peptide determination as a screening test for the detection of patients with mild left ventricular impairment, Heart 76(3) (1996), 232-237. 
[37] A. Peer, G. Falkensammer, H. Alber et al., Limited utilities of $\mathrm{N}$-terminal pro B-type natriuretic peptide and other newer risk markers compared with traditional risk factors for prediction of significant angiographic lesions in stable coronary artery disease, Heart 95(4) (2009), 297-303.

[38] C. Kragelund, B. Groenning, T. Omland et al., Is N-terminal pro B-type natriuretic peptide (NT-proBNP) a useful screening test for angiographic findings in patients with stable coronary disease? Am Heart J 151 (2006), 712.e1-712.e7.

[39] L. Amato, G. Paolisso, F. Cacciatore et al., Congestive heart failure predicts the development of non- insulindependent di- abetes mellitus in the elderly.The Osservatorio Geriatrico Regione Campania Group, Diabetes Metab 23 (1997), 213-218.

[40] M.H. Olsen, T.W. Hansen, M.K. Christensen et al., N-terminal pro brain natriuretic peptide is inversely related to metabolic cardiovascular risk factors and the metabolic syndrome, $\mathrm{Hy}$ pertension 46 (2005), 660-666.

[41] P. Hildebrandt, M. Boesen, M. Olsen et al., N-terminal pro brain natriuretic peptide in arterial hypertension - a marker or left ventricular dimensions and prognosis, Eur J Heart Fail 6 (2004), 313-317. 


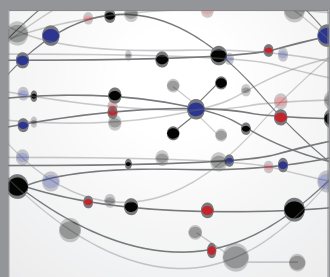

The Scientific World Journal
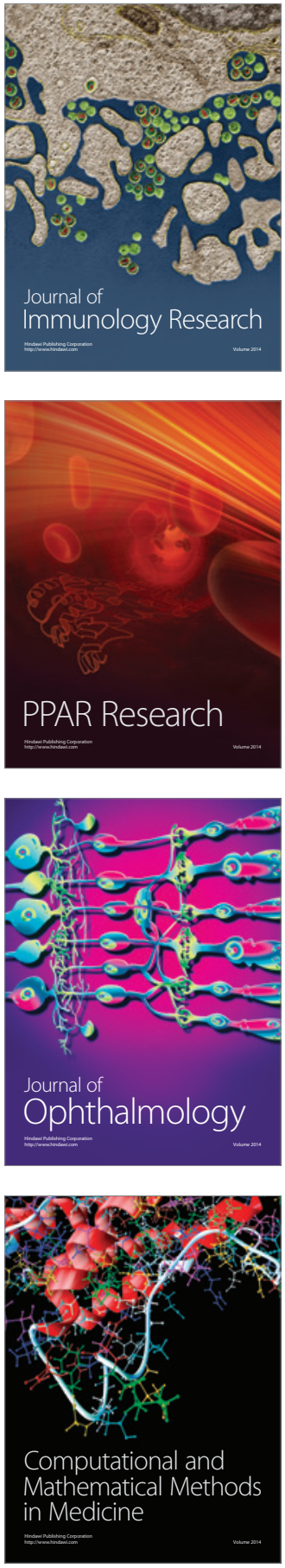

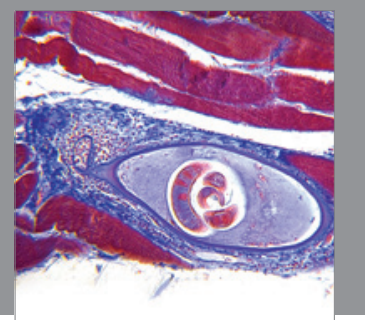

Gastroenterology

Research and Practice
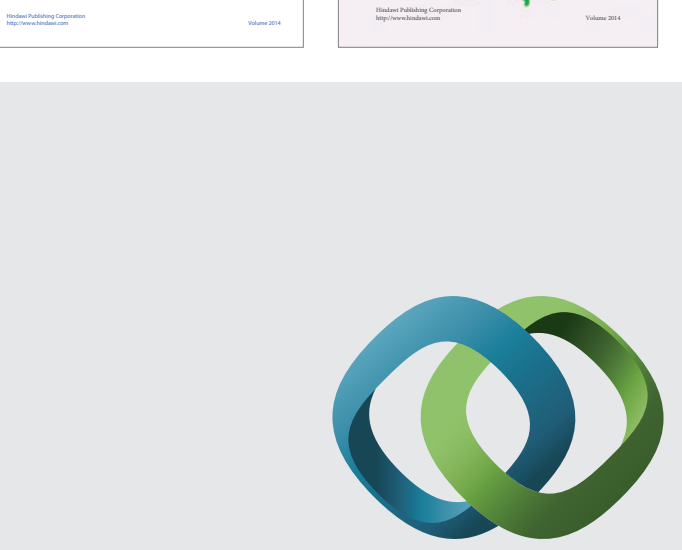

\section{Hindawi}

Submit your manuscripts at

http://www.hindawi.com
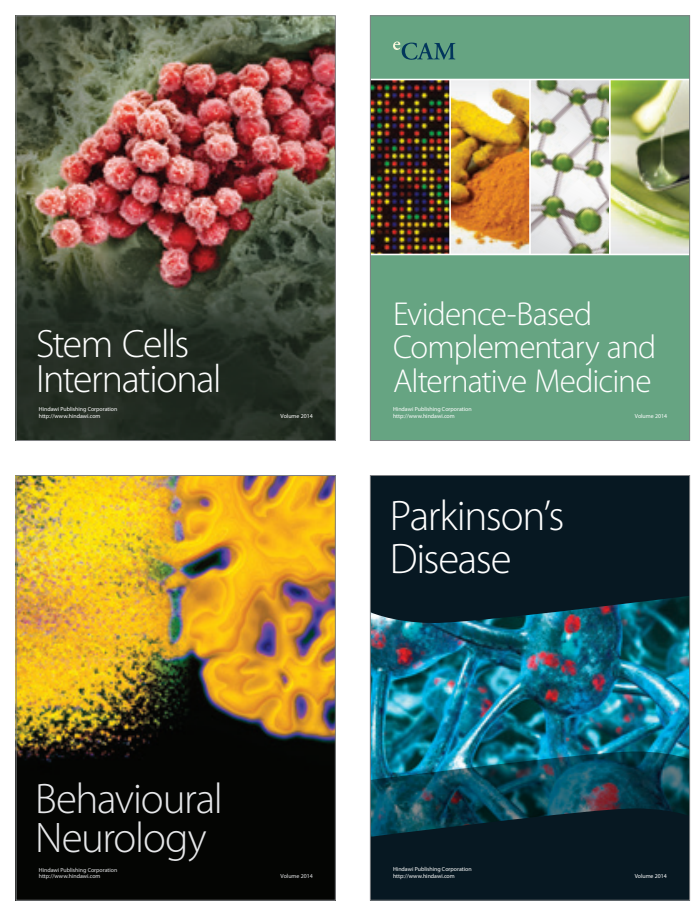

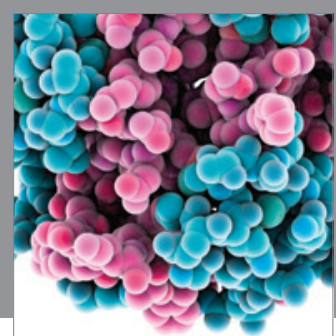

Journal of
Diabetes Research

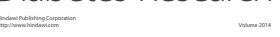

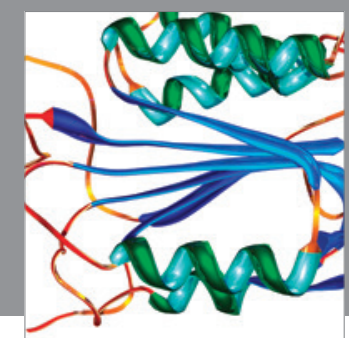

Disease Markers
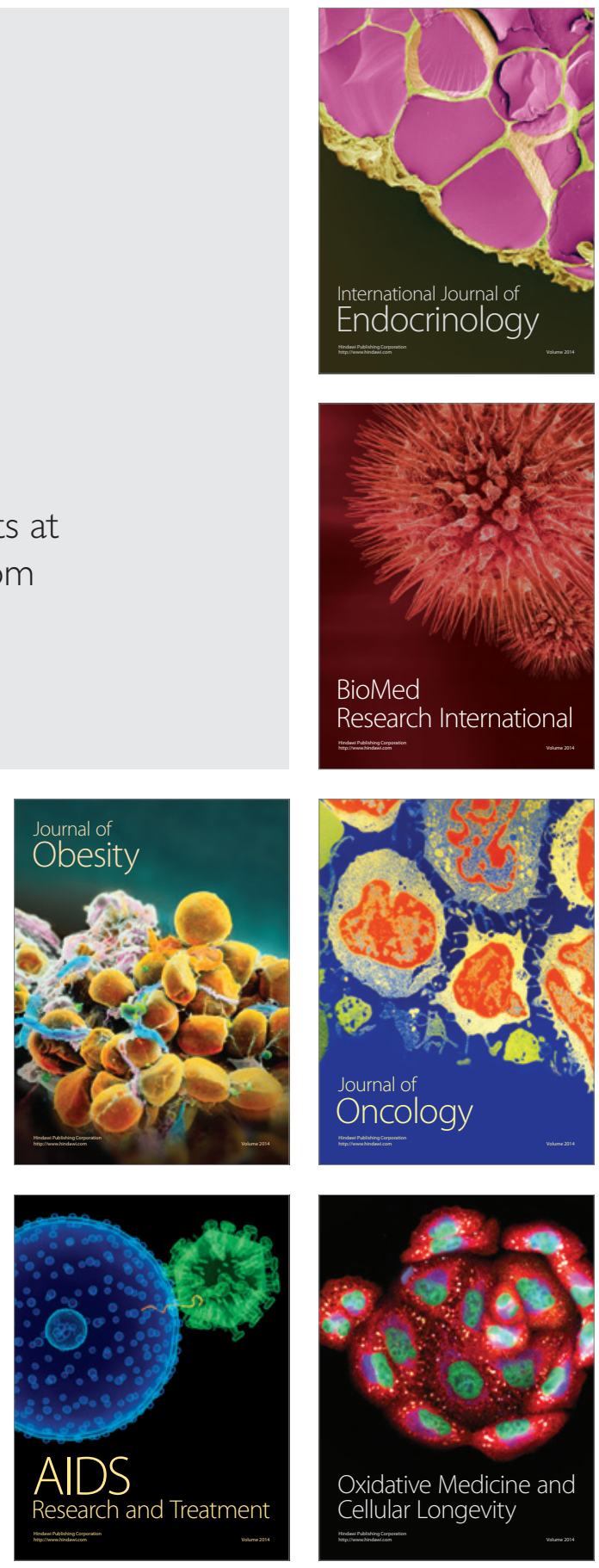